Pacific Journal of Mathematics

A CHARACTERIZATION OF THE MAXIMAL MONOIDS AND 


\title{
A CHARACTERIZATION OF THE MAXIMAL MONOIDS AND MAXIMAL GROUPS IN $\beta_{X}$
}

\author{
D. J. Hartfiel and C. J. Maxson
}

This paper gives a characterization of the maximal monoids and maximal groups in $\beta_{X}$, the semigroup of binary relations. The characterization is applied to show that the maximal group containing the partial order $\alpha$ is isomorphic to the group of order automorphisms of $(X, \alpha)$.

Introduction. In establishing some background research in which the setting of this current paper can be placed, one cites that much work has been done on the semigroup $\beta_{X}$, the binary relations on the set $X$. In particular, the works of Montague and Plemmons [4], Plemmons and Schein [5], Schwarz [6], as well as Clifford [3] appear dominant in the study of the maximal groups contained in $\beta_{X}$. The basic result of these papers shows that the maximal group of $\beta_{X}$ containing the partial order $\alpha$, is isomorphic to Auto $(X, \alpha)$, the group of order automorphisms of $(X, \alpha)$. Thus, as Birkhoff's Theorem provides that every group is isomorphic to a group of automorphisms on some partially ordered set $(X, \alpha)$, it follows that every group is isomorphic to a maximal group in $\beta_{X}$, for some $X$.

The work of this paper is concerned with maximal monoids as well as maximal groups in $\beta_{X}$. The work differs from previously published papers in that the concern here is to provide a unified characterization of these two algebraic systems of $\beta_{X}$. The characterization then provides a natural avenue to obtain the isomorphism theorems of Montague and Plemmons [4], Plemmons and Schein [5], and Clifford [3]. Further, by viewing the isomorphism theorems through our characterization, the researcher obtains some measure of intuition as to their validity.

As a convenient method of presentation, the paper is divided into two parts, a section of genral results, followed by a section of applications in which our general results are applied to $\beta_{X}$ for $X$ infinite and then $X$ finite.

General results. Let $\alpha \in \beta_{X}$. We associate with $\alpha$ a Boolean matrix whose $(x, y)$ th entry is 1 if and only if $(x, y) \in \alpha$. This correspondence provides an isomorphism between the semigroup $\beta_{X}$ and the semigroup $B_{X}$ of $X \times X$ Boolean matrices. Thus, without loss of generality, we characterize the maximal monoids and maximal group in $B_{X}$. 
Let $V_{X}$ be the collection of $X \times 1$ Boolean vectors. If $A \in B_{X}, A^{x}$ denotes the $x$ th column of $A$. By $z=\sum_{x \in \tau} A^{x}$ we mean the vector in $V_{X}$ with the property that the $y$ th entry in $z$ is 1 if and only if there is an $x \in \tau$ such that $A^{x}$ has $y$ th entry 1. Set $R(A)=\left\{A x \mid x \in V_{X}\right\}$. By End $R(A)$ we mean all cone endomorphisms from $R(A)$ into $R(A)$, i.e. $\left\{\psi \mid \psi\left(\sum_{x \in \tau} a_{x} z^{x}\right)=\sum_{x \in \tau} a_{x} \psi\left(z^{x}\right)\right.$ where $z^{x} \in R(A)$ and $a_{x} \in B, B$ the Boolean algebra, for all $x \in \tau$, and index set\}. Similarly, Aut $R(A)=$ $\{\psi \in$ End $R(A) \mid \psi$ is one-to-one and onto $\}$.

It should be noted here that $\psi(a x+b y)=a \psi(x)+b \psi(y)$ for all $x, y \in R(A)$ and $a, b \in B$ does not guarantee $\psi \in$ End $R(A)$. For example, let $X=\{1,2, \cdots, n, \cdots\}$. Define

$\psi(x)=x$ if $x$ has only finitely many nonzero entries, and

$\psi(x)=e=(1,1, \cdots, 1, \cdots)^{t}$ if $x$ has infinitely many nonzero entries. Thus $\psi(a x+b y)=a \psi(x)+b \psi(y)$ for all $x, y \in V_{X}$ and $a, b \in B$. Now let $e_{i} \in V_{X}$ be the vector with precisely one 1 , which is in the $i$ th position. Set $z=\sum_{i=1}^{\infty} e_{2 i}$. Then $\psi(z)=e$, yet $\sum_{i=1}^{\infty} \psi\left(e_{2 i}\right)=\sum_{i=1}^{\infty} e_{2 i} \neq e$, and hence $\psi \notin$ End $R(I)$ where $I$ is the identity matrix.

Finally, if $I \in B_{X}$ is an idempotent,

$$
M(I)=\left\{A \in B_{X} \mid A I=I A=A\right\}
$$

and

$$
G(I)=\{\text { units in } M(I)\}
$$

$M(I)$ is the maximal monoid with identity $I$ and of course, $G(I)$ is the maximal group in $B_{X}$ which contains $I$.

For further background in this area, the reader is referred to [1].

Our initial results characterize $M(I)$.

Lemma 1. Set $z=\sum_{x \in \tau} z^{x}$ where $z^{x} \in V_{X}$ for $x \in \tau$. If $A \in B_{X}$, $A z=\sum_{x \in \tau} A z^{x}$.

Proof. For $w \in V_{X}$, let $w_{y}$ denote the $y$ th entry in $w$. Now $z_{y}=1$ if and only if $z_{y}^{x}=1$ for some $x \in \tau$. By calculation,

$$
A z=\sum_{z_{y}=1} A^{y}=\sum_{\substack{z_{y}=1 \\ x \in \tau}} A^{y}=\sum_{x \in \tau} A z^{x}
$$

Hence the lemma follows.

Our characterization of $M(I)$ is contained in the next theorem.

TheOREM 1. End $R(I)$ is isomorphic to $M(I)$ for any idempotent $I \in B_{X}$. 
Proof. For each $\psi \in$ End $R(I)$, consider the matrix $A_{\psi}$ whose $x$ th column is $\psi\left(I^{x}\right)$. Pick $\sigma \in$ End $R(I)$. Then $A_{\psi} \cdot A_{\sigma}$ has $x$ th column $\Sigma_{y}\left[\sigma\left(I^{x}\right)\right]_{y} \psi\left(I^{y}\right)$ where $\left[\sigma\left(I^{x}\right)\right]_{y}$ is the $y$ th entry of $\sigma\left(I^{x}\right)$. Now

$$
\begin{aligned}
\sum_{y}\left[\sigma\left(I^{x}\right)\right]_{y} \psi\left(I^{y}\right) & =\psi\left[\sum_{y}\left[\sigma\left(I^{x}\right)\right]_{y} I^{y}\right]=\psi\left[\operatorname{I\sigma }\left(I^{x}\right)\right] \\
& =\psi\left[\sigma\left(I^{x}\right)\right]=\psi \circ \sigma\left(I^{x}\right) .
\end{aligned}
$$

Thus $A_{\psi} A_{\sigma}=A_{\psi \circ \sigma}$.

Now, if 1 is the identity endomorphism, $A_{1}=I$ and so $A_{\psi} I=I A_{\psi}=$ $A_{\psi}$ from which it follows that $A_{\psi} \in M(I)$.

Consider the map $\pi(\psi)=A_{\psi}$ of End $R(I)$ into $M(I)$. Of course $\pi$ is a monomorphism. To show $\pi$ is onto, pick $A \in M(I)$ and set $\psi(z)=A z$ for each $z \in R(I)$. Then by Lemma $1, \quad \psi \in$ End $R(I)$. Since $A I=A$, the $x$ th column of $A$ is $\psi\left(I^{x}\right)$ and hence $A=A_{\psi}$.

CoRollary 1. Aut $R(I)$ is isomorphic to $G(I)$ for any idempotent $I \in B_{X}$.

Proof. The group of units of $M(I)$ is $G(I)$ while the group of units of End $R(I)$ is Aut $R(I)$. Since a monoid isomorphism maps units to units the result follows.

We say that a set $\mathscr{S}=\left\{S^{x} \mid S^{x} \in V_{X}\right.$ for $x \in T$, some index set $\}$ is independent if and only if no $S^{x}=0$ and

$$
\sum_{x \in N \subseteq T} S^{x}=S^{y} \quad \text { implies } \quad y \in N .
$$

Further, if $w, z \in V_{X}$ we say $w \leqq z$ if and only if the $x$ th entry of $z$ being 1 implies the $x$ th entry of $w$ is 1 . Note that $\Sigma_{x \in N \varsigma_{\tau}} S^{x} \geqq S^{x}$ for each $x \in N$ and thus in independence proofs one needs only to check that $S^{y}=\sum_{x \in N \subseteq \tau} S^{x}$ implies $S^{y} \leqq S^{x}$ for some $x \in N$. For $S, T \subseteq V_{X}$ we say that $\psi$ is an order map from $S$ to $T$ if and only if for $w \leqq z$ in $S, \psi(w) \leqq \psi(z)$ in $T$.

With these definitions, our direction of research is now to refine the characterization of $M(I)$ and thereby to provide a link from Aut $R(I)$ to Auto $(X, I)$. The instrument for this link is given in the next theorem.

Theorem 2. Let $S \in B_{X}$ and $\mathscr{S}=\left\{S^{x} \mid x \in \tau\right.$, $\tau$ some index set $\}$ be independent such that

(1) $R(S)=\left\{w \mid w=\Sigma_{x \in N \subseteq \tau} S^{x}\right\} \cup\{0\}$.

(2) $\sum_{x \in N \subseteq \tau} S^{x} \geqq S^{y}$ implies some $S^{x} \geqq S^{y}, x \in N$. 
Then if $\psi$ is an order map from $\mathscr{S}$ into $R(S)$ there is a unique $\bar{\psi} \in$ End $R(S)$ so that $\bar{\psi}=\psi$ on $\mathscr{S}$.

Proof. Define $\bar{\psi}\left(S^{x}\right)=\psi\left(S^{x}\right)$ for each $x \in \tau$.

$$
\begin{aligned}
& \bar{\psi}(0)=0 . \\
& \bar{\psi}\left(\sum_{x \in N \subseteq \tau} S^{x}\right)=\sum_{x \in N \subseteq \tau} \psi\left(S^{x}\right) .
\end{aligned}
$$

To see that $\bar{\psi}$ is well defined, note that if $\Sigma_{x \in N{ }_{\tau}} S^{x}=\Sigma_{x \in M \varsigma_{\tau}} S^{x}$ then for each $y \in M$ there is an $x \in N$ so that $S^{x} \geqq S^{y}$. Thus $\bar{\psi}\left(S^{x}\right)=\psi\left(S^{x}\right) \geqq$ $\psi\left(S^{y}\right)=\bar{\psi}\left(S^{y}\right)$ and hence $\bar{\psi}\left(\sum_{x \in N} S^{x}\right) \geqq \bar{\psi}\left(\sum_{x \in M} S^{x}\right)$. Similarly, $\bar{\psi}\left(\sum_{x \in M} S^{x}\right) \geqq \bar{\psi}\left(\sum_{x \in N} S^{x}\right)$ and so $\bar{\psi}\left(\sum_{x \in N} S^{x}\right)=\bar{\psi}\left(\sum_{x \in M} S^{x}\right)$.

To see that $\bar{\psi} \in$ End $R(S)$ pick $z^{x} \in R(S), x \in N$ where $N$ is some index set. Suppose $z^{x}=\Sigma_{y \in N_{x}} S^{y}, N_{x}$ an index set. Consider

$$
\begin{aligned}
\bar{\psi}\left(\sum_{x \in N} z^{x}\right)=\bar{\psi}\left(\sum_{\substack{y \in N_{x} \\
x \in N}} S^{y}\right) & =\sum_{\substack{y \in N_{x} \\
x \in N}} \psi\left(S^{y}\right)=\sum_{x \in N} \sum_{y \in N_{x}} \psi\left(S^{y}\right) \\
& =\sum_{x \in N} \bar{\psi}\left(\sum_{x \in N_{x}} S^{y}\right)=\sum_{x \in N} \bar{\psi}\left(z^{x}\right) .
\end{aligned}
$$

Thus $\bar{\psi} \in$ End $R(S)$.

Finally, the uniqueness of $\bar{\psi}$ is clear as any $\sigma \in \operatorname{End} R(S)$ must satisfy $\sigma\left(\Sigma_{x \in M \subseteq T} S^{x}\right)=\Sigma_{x \in M \subseteq T} \sigma\left(S^{x}\right)$ and hence is uniquely determined on the independent set.

We now apply the results of this section to illustrate the utility of our characterization and to obtain the isomorphism theorem of Montague, Plemmons, Schein, and Clifford, as a consequence.

Applications. The organization of this section is to separate the applications of the general results to $B_{X}$ into two cases, i.e. $X$ infinite and $X$ finite.

Case 1. $X$ infinite. Our first lemma shows that a partial order satisfies the hypotheses of Theorem 2 .

LeMmA 2. If $I$ is a partial order, $\mathscr{S}=\left\{I^{x} \mid x \in X\right\}$ is independent with $\sum_{x \in N \subseteq X} I^{x} \geqq I^{y}$ implying some $I^{x} \geqq I^{y}$ for $x \in N$.

Proof. Let $I^{y} \in \mathscr{S}$ such that $I^{y}=\Sigma_{x \in N \subseteq X} I^{x}$. As $(y, y) \in I$, $(y, x) \in I$ for some $x \in N$. Now $(z, y) \in I$ implies $(z, x) \in I$. So $I^{x} \geqq$ $I^{y}$ and hence $\mathscr{S}$ is independent.

Now consider 


$$
\sum_{x \in N \subseteq X} I^{x} \geqq I^{y} \quad \text { for some } y .
$$

Then as in the above argument, there is some $x \in N$ so that $I^{x} \geqq$ $I^{y}$. Hence the result follows.

From this lemma, we can argue our first isomorphism theorem.

THEOREM 3. If $I$ is a partial order, Aut $R(I)$ is isomorphic to Auto $(\mathscr{S}, \leqq)$ where $\mathscr{S}=\left\{I^{x} \mid X \in X\right\}$.

Proof. By Lemma 2, $\mathscr{S}$ satisfies the hypothesis of Theorem 2. Thus, any $\psi \in$ Auto $(\mathscr{S}, \leqq)$ extends to a unique $\bar{\psi} \in$ Aut $R(I)$. Conversely, any $\bar{\psi} \in$ Aut $R(I)$, when restricted to $\mathscr{S}$, determines a $\psi \in \operatorname{Auto}(\mathscr{S}, \leqq)$. Thus Auto $(\mathscr{S}, \leqq)$ is isomorphic to Aut $R(I)$.

The Plemmons, Schein, Clifford result is an immediate application.

Corollary 3. If $I$ is a partial order, $G(I)$ is isomorphic to $\operatorname{Auto}(X, I)$.

Proof. From previous results, $G(I)$ is isomorphic to Auto $(\mathscr{S}, \leqq)$, $\mathscr{S}=\left\{I^{x} \mid x \in X\right\}$. Finally, note that $(x, y) \in I$ if and only if $I^{x} \leqq$ $I^{y}$. Thus Auto $(\mathscr{S}, \leqq)$ is isomorphic to Auto $(X, I)$.

To illustrate the utility of our characterization, we provide the following example.

EXAMPLE. Let $X=[0,1]$ and let $I$ be the natural partial order $\geqq$ on $X$. Clearly, $\left\{I^{x} \mid x \in X\right\}$ is independent. Further, $R(I)=$ $\left\{I^{x} \mid x \in X\right\}=\mathscr{S}$ and hence $\{\psi \mid \psi: \mathscr{S} \rightarrow R(I), \psi$ an order map $\}=$ $\{\psi \mid \psi: \mathscr{S} \rightarrow \mathscr{S}, \psi$ an order map $\}$. Thus, it follows that $M(I)$ is isomorphic to the semigroup of nondecreasing functions $f: X \rightarrow X$ so that $f(0)=0$. Hence $G(I)$ is the group of continuous strictly increasing functions $f: X \rightarrow X$, so that $f(0)=0$ and $f(1)=1$.

Case 2. $X$ finite. For this case we use the following result of Schwarz [7].

Idempotent Theorem. If $I$ is an idempotent then there is a permutation matrix $P$ so that 


$$
P^{t} I P=\left[\begin{array}{lllll}
A_{1} & 0 & \cdots & 0 & 0 \\
A_{21} & A_{2} & \cdots & 0 & 0 \\
& & & & \\
A_{s-1,1} & A_{s-1,2} & \cdots & A_{s-1} & 0 \\
A_{s, 1} & A_{s, 2} & \cdots & A_{s, s-1} & A_{s}
\end{array}\right]_{\text {where }}
$$
order one,

(1) each $A_{k}$ is composed entirely of 1's or $A_{k}=(0)$, the 0-matrix of

(2) each $A_{k j}$ is composed entirely of 1's or entirely of 0's,

then $a_{k} \geqq a_{j}$,

if $A_{j k}>0$ and $a_{k}$ a column in $\left[\begin{array}{c}0 \\ \vdots \\ 0 \\ A_{k} \\ \vdots \\ A_{s, k}\end{array}\right]$, a column in $\left[\begin{array}{c}0 \\ \vdots \\ 0 \\ A_{i} \\ \vdots \\ A_{s, j}\end{array}\right]$,

(4) if $A_{i}=A_{j}=(0), i>j$, and $A_{i j} \neq(0)$ then there is a $k, i>k>j$ so that $A_{k}, A_{i k}$ and $A_{k j}$ are composed entirely of 1 's.

Let $I$ be a nonzero finite idempotent. Without loss of generality we assume $I$ has the form given in the Idempotent theorem. As a consequence of this theorem we note that $I$ has a nonzero main diagonal element and, in fact the next theorem shows that this characterizes the independent columns of $I$.

To show that the situation is different for infinite idempotents consider the set $X=[0,1]$ and define $I \in B_{X}$ by $(x, y) \in I$ if and only if $x>y$. Then $I$ is an idempotent with no set of independent columns that generate $R(I)$ and $I$ has no nonzero diagonal elements.

THEOREM 4. Let I be a finite nonzero idempotent and $\mathscr{S}$ the set of distinct vectors $I^{x}$ such that $(x, x) \in I$. Then $\mathscr{S}$ is an independent set satisfying the conditions of Theorem 2.

Proof. From the remarks above, $\mathscr{S} \neq \varnothing$. Clearly $\mathscr{S}$ is independent and satisfies Condition 2. Thus, we need only prove $R(I)=$ $\left\{w \mid w=\Sigma_{I^{x} \in N \mathcal{S}^{\mathscr{S}}} I^{x}\right\} \cup\{0\}$. Let $I^{y}$ be nonzero and not in $\mathscr{S}$. Let $T=$ $\left\{I^{x} \mid(x, x) \in I\right.$ and $\left.(x, y) \in I\right\}$. From (4) of the Idempotent Theorem, $T \neq \varnothing$. We show $\Sigma_{T} I^{x}=I^{y}$. It is seen from (3) of the Idempotent Theorem that $I^{y} \geqq I^{x}$ for all $I^{x} \in T$. Thus pick any $(z, y) \in$ I. Consider $I^{z}$. If $(z, z) \in I$ then $I^{z} \in T$. If $(z, z) \notin I$, then by (4) of the Idempotent Theorem there is an $I^{x}, y<x<z$ with $(x, x) \in I$ and $(z, x) \in I$. Hence, $(z, x) \in \Sigma_{T} I^{x}$ and the result follows. 
We now give the finite analogue of Theorem 3. Note that in this case, the partial order assumption is not required. Indeed the result holds for any idempotent.

THEOREM 5. If $I$ is a finite nonzero idempotent, Aut $R(I)$ is isomorphic to Auto $(\mathscr{S} \leqq)$ where $\mathscr{S}$ is as given in Theorem 4 .

The Montague, Plemmons result follows as a consequence.

COROllary 4. If $I$ is a finite partial order, $G(I)$ is isomorphic to Auto $(X, I)$.

In conclusion, we provide an example indicating how our characterization theorem can be applied for the case $X$ is finite.

Example. Consider the idempotent

$$
I=\left[\begin{array}{lll}
1 & 0 & 0 \\
1 & 0 & 0 \\
1 & 0 & 1
\end{array}\right]
$$

Then

$$
R(I)=\left\{\left(\begin{array}{l}
1 \\
1 \\
1
\end{array}\right),\left(\begin{array}{l}
0 \\
0 \\
0
\end{array}\right),\left(\begin{array}{l}
0 \\
0 \\
1
\end{array}\right)\right\}
$$

with

$$
\left(\begin{array}{l}
1 \\
1 \\
1
\end{array}\right),\left(\begin{array}{l}
0 \\
0 \\
1
\end{array}\right) \text { independent and }\left(\begin{array}{l}
1 \\
1 \\
1
\end{array}\right) \geqq\left(\begin{array}{l}
0 \\
0 \\
1
\end{array}\right)
$$

Hence, using Theorem 2,

$$
M(I)=\left(I,[0],\left[\begin{array}{lll}
1 & 0 & 1 \\
1 & 0 & 1 \\
1 & 0 & 1
\end{array}\right],\left[\begin{array}{lll}
0 & 0 & 0 \\
0 & 0 & 0 \\
1 & 0 & 1
\end{array}\right],\left[\begin{array}{lll}
1 & 0 & 0 \\
1 & 0 & 0 \\
1 & 0 & 0
\end{array}\right],\left[\begin{array}{lll}
0 & 0 & 0 \\
0 & 0 & 0 \\
1 & 0 & 0
\end{array}\right]\right\}
$$

and

$$
G(I)=\{I\}
$$

Other such examples are easily constructed. 


\section{REFERENCES}

1. J. C. Abbott, Sets Lattices and Boolean Algebras, Allyn and Bacon Inc., Boston, 1969.

2. G. Birkhoff, Sobre los grupos de automorfismos, Rev. Un. Math., Argentina, 11 (1964), 155-157.

3. A. H. Clifford, A proof of the Montague-Plemmons-Schein theorem of maximal subgroups of a semigroup of binary relations, Semigroup Forum, 1 (1970), 272-275.

4. J. S. Montague and R. J. Plemmons, Maximal subgroups of the semigroup of relations, J. of Algebra, 13 (1969), 575-587.

5. R. J. Plemmons and B. M. Schein, Groups of binary relations, Semigroup Forum, 1 (1970), $267-271$.

6. Stefan Schwarz, The semigroup of binary relations on a finite set, presented to the International Symposium on Semigroups, Bratislava, Czecheslovakia, June, 1968.

7. - On idempotent binary relations on a finite set, Czechoslavak Math. J. 20 (1970), 696-702.

Received May 16, 1974.

TEXAS A\&M UNIVERSITY 


\section{PACIFIC JOURNAL OF MATHEMATICS}

\section{EDITORS}

RICHARD ARENS (Managing Editor)

University of California

Los Angeles, California 90024

\section{J. DugundJI}

Department of Mathematics University of Southern California Los Angeles, California 90007

D. Gilbarg and J. Milgram

Stanford University

Stanford, California 94305

\section{ASSOCIATE EDITORS}
E. F. BECKENBACH
B. H. NeumanN
F. WoLF
K. YoshiDA

\section{SUPPORTING INSTITUTIONS}

\author{
UNIVERSITY OF BRITISH COLUMBIA \\ CALIFORNIA INSTITUTE OF TECHNOLOGY \\ UNIVERSITY OF CALIFORNIA \\ MONTANA STATE UNIVERSITY \\ UNIVERSITY OF NEVADA \\ NEW MEXICO STATE UNIVERSITY \\ OREGON STATE UNIVERSITY \\ UNIVERSITY OF OREGON \\ OSAKA UNIVERSITY
}

\author{
UNIVERSITY OF SOUTHERN CALIFORNIA \\ STANFORD UNIVERSITY \\ UNIVERSITY OF TOKYO \\ UNIVERSITY OF UTAH \\ WASHINGTON STATE UNIVERSITY \\ UNIVERSITY OF WASHINGTON \\ AMERICAN MATHEMATICAL SOCIETY
}

The Supporting Institutions listed above contribute to the cost of publication of this Journal, but they are not owners or publishers and have no responsibility for its contents or policies.

Mathematical papers intended for publication in the Pacific Journal of Mathematics should be in typed form or offset-reproduced (not dittoed), double spaced with large margins. Underline Greek letters in red, German in green, and script in blue. The first paragraph or two must be capable of being used separately as a synopsis of the entire paper. Items of the bibliography should not be cited there unless absolutely necessary, in which case they must be identified by author and Journal, rather than by item number. Manuscripts, in duplicate, may be sent to any one of the four editors. Please classify according to the scheme of Math. Reviews, Index to Vol. 39. All other communications should be addressed to the managing editor, or Elaine Barth, University of California, Los Angeles, California, 90024.

100 reprints are provided free for each article, only if page charges have been substantially paid. Additional copies may be obtained at cost in multiples of 50 .

The Pacific Journal of Mathematics is issued monthly as of January 1966. Regular subscription rate: $\$ 72.00$ a year (6 Vols., 12 issues). Special rate: $\$ 36.00$ a year to individual members of supporting institutions.

Subscriptions, orders for back numbers, and changes of address should be sent to Pacific Journal of Mathematics, 103 Highland Boulevard, Berkeley, California, 94708.

PUBLISHED BY PACIFIC JOURNAL OF MATHEMATICS, A NON-PROFIT CORPORATION Printed at Jerusalem Academic Press, POB 2390, Jerusalem, Israel.

$$
\begin{gathered}
\text { Copyright (C) } 1975 \text { Pacific Journal of Mathematics } \\
\text { All Rights Reserved }
\end{gathered}
$$




\section{Pacific Journal of Mathematics}

\section{Vol. 58, No. $2 \quad$ April, 1975}

Zvi Artstein and John Allen Burns, Integration of compact set-valued functions . . . . . . . . . 297

Mark Benard, Characters and Schur indices of the unitary reflection group $[321]^{3} \ldots \ldots \ldots . .309$

Simeon M. Berman, A new characterization of characteristic functions of absolutely continuous

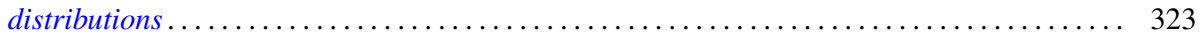

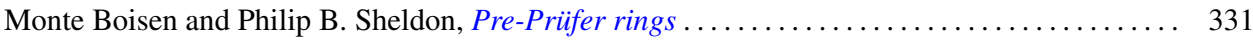

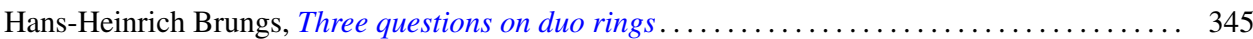

Iracema M. Bund, Birnbaum-Orlicz spaces of functions on groups................. 351

John D. Elwin and Donald R. Short, Branched immersions between 2-manifolds of higher

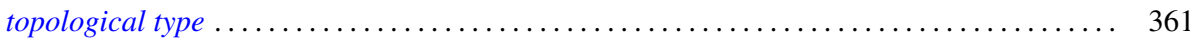

Eric Friedlander, Extension functions for rank 2, torsion free abelian groups . .......... 371

Jon Froemke and Robert Willis Quackenbush, The spectrum of an equational class of

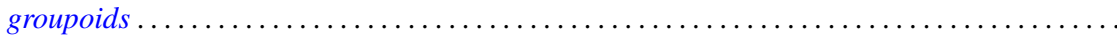

Barry J. Gardner, Radicals of supplementary semilattice sums of associative rings ...........

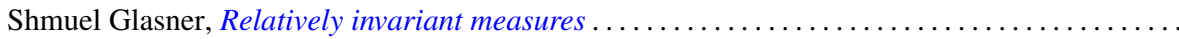

George Rudolph Gordh, Jr. and Sibe Mardesic, Characterizing local connectedness in inverse

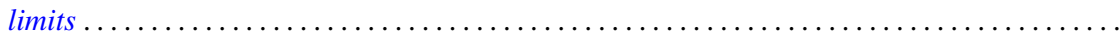

Siegfried Graf, On the existence of strong liftings in second countable topological spaces......

Stanley P. Gudder and D. Strawther, Orthogonally additive and orthogonally increasing

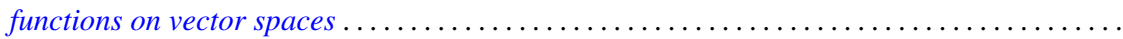

Darald Joe Hartfiel and Carlton James Maxson, A characterization of the maximal monoids and

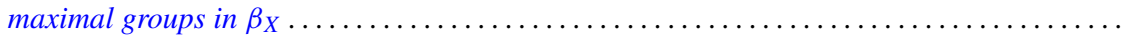

Robert E. Hartwig and S. Brent Morris, The universal flip matrix and the generalized faro-shuffle. .

William Emery Haver, Mappings between ANRs that are fine homotopy equivalences. .

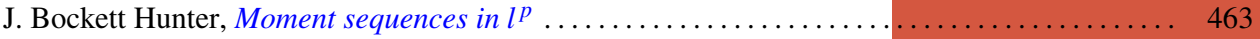

Barbara Jeffcott and William Thomas Spears, Semimodularity in the completion of a poset.... 467

Jerry Alan Johnson, A note on Banach spaces of Lipschitz functions . . . . . . . . . . . . 475

David W. Jonah and Bertram Manuel Schreiber, Transitive affine transformations on

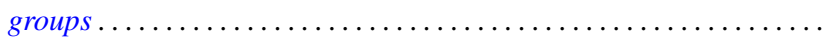

Karsten Juul, Some three-point subset properties connected with Menger's characterization of

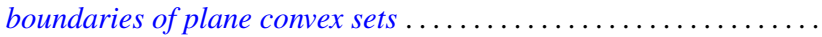

Ronald Brian Kirk, The Haar integral via non-standard analysis . . . . . . . . . . . . . 517

Justin Thomas Lloyd and William Smiley, On the group of permutations with countable

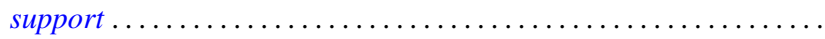

Erwin Lutwak, Dual mixed volumes .................................. 531

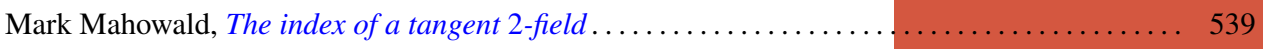

Keith Miller, Logarithmic convexity results for holomorphic semigroups . . . . . . . . . . . . 549

Paul Milnes, Extension of continuous functions on topological semigroups . . . . . . . . . . 553

Kenneth Clayton Pietz, Cauchy transforms and characteristic functions ................ 563

James Ted Rogers Jr., Whitney continua in the hyperspace $C(X) \ldots \ldots \ldots \ldots \ldots \ldots \ldots \ldots .569$

Jean-Marie G. Rolin, The inverse of a continuous additive functional . . . . . . . . . . . . 585

William Henry Ruckle, Absolutely divergent series and isomorphism of subspaces . ........ 605

Rolf Schneider, A measure of convexity for compact sets . ..................... 617

Alan Henry Schoenfeld, Continous measure-preserving maps onto Peano spaces .......... 627

V. Merriline Smith, Strongly superficial elements .......................... 643

Roger P. Ware, A note on quadratic forms over Pythagorean fields . . . . . . . . . . . . . . 651

Roger Allen Wiegand and Sylvia Wiegand, Finitely generated modules over Bezout rings . . . . 655

Martin Ziegler, A counterexample in the theory of definable automorphisms . . . . . . . . . 665 